\title{
Virtual Crossmatch in the Context of Kidney Transplantation
}

\author{
Priscila de Moraes, Beatriz Chamun Gil* and Luiz Fernando Jobim \\ Department of serology, Hospital de Clínicas de Porto Alegre, Brasil \\ *Corresponding author: Beatriz Chamun Gil, Department of serology, Hospital de Clínicas de Porto Alegre, Ramiro Barcelos, 2350 - 90035903 , \\ Porto Alegre, Rio Grande do Sul, Brasil
}

Submission: 眥April 11, 2018; Published: 眥 May 11, 2018

Keywords: Virtual crossmatch; Donor specific antibodies; Human leukocyte antigen

Abbreviations: VXM: Virtual Crossmatch; CDC: Complement Dependent Cytotoxicity; FCXM: Flow Cytometric Crossmatch; HLA: Human Leukocyte Antigen; SA: Single Antigen; DSA: Donor Specific Antibodies; MFI: Mean Fluorescence Intensity

\section{Introduction}

The patient survival is strongly influenced by the presence of DSA [1-5]. Currently, Virtual Cross Match (VXM) has been used to predict the real crossmatch results by Complement-Dependent Cytotoxicity (CDC) and Flow Cytometry crossmatch (FCXM) [6-10]. VXM compare donor HLA antigens with anti-HLA antibodies in recipient serum. The antibodies are detected by the Single Antigen (SA) assay, in which each bead is coated with a single HLA specificity, detecting the precise anti-HLA antibodies and their levels, allowing predict reactions against donor HLA antigens. However, SA assays has some limitations that can, difficult the VXM interpretation [11]. The SA assay show higher sensitivity whereas the CDC and FCXM are specific against donor HLA molecules, which suggests that methods may be complementary [7-10].

\section{Discussion}

The high sensitivity and specificity of the SA assay in the detection of DSAs allows for the performance of a virtual crossmatch, although many studies have demonstrated that virtual crossmatch has limited value in predicting the result of the crossmatch, because the SA assay has some disadvantages [10-15]. The concentration of HLA in beads may vary due to differences in the binding of HLA molecules to the bead surface, not representing the antigens present in graft tissue. SA assay is not donor specific and use purified HLA antigens bound to the beads, thus possibly distorting molecular conformation during the binding process and can provide a false positive result and SA bead panel cannot include rare HLA antigens [11-16]. Further, the HLA typing of donors performed on HLA-A, B, C, DR, DQ and DP loci shows the importance for the accurate assessment of the presence of DSA. Previous studies have demonstrated that DSA with median fluorescence intensity $(\mathrm{MFI}) \geq 5000$ correlate with FCXM and CDC positive, this cut off has been commonly used in VXM[10,12]. However, some publications indicate that MFI, although is a numerical value, should not be used to quantify antibodies $[11,17]$.

In this context, use a fixed cutoff for VXM may not be an adequate option, in fact there is no single value that corresponds to a threshold above or below which contraindicated the transplantation without risk [18]. VXM allow for the quantification of antibodies by MFI and patient stratification by risk groups (sensitization), therefore it should be used with aim of favoring hyper sensitized patients and not excluding them from being tested in real crossmatch (CDC e FCXM) [19], which suggests that the methods are complementary[8,20,21].

\section{Conclusion}

The antibodies detection techniques are increasingly sensitive and specific, however, the excess of information can difficult to interpretation results and make decisions. The analysis of the techniques together (CDC, FCXM and VXM) as well as the patient historic, can provide important information to transplantation even on the presence of DSA and may help in the post-transplant patient monitoring and in immunosuppressive strategy.

\section{References}

1. Singh N, Djamali A, Lorentzen D, Pirsch JD, Leverson G, et al. (2010) Pretransplant Donor-Specific Antibodies Detected by Single-Antigen Bead Flow Cytometry Are Associated With Inferior Kidney Transplant Outcomes. Transplantation 90(10): 1079-1084.

2. Patel AM, Pancoska C, Mulgaonkar S, Weng FL ( 2007) Renal transplantation in patients with pre-transplant donor-specific antibodies and negative flow cytometry crossmatches. Am J Transplant $7(10):$ 2371-2377.

3. Terasaki PI, Ozawa M (2004) Predicting Kidney Graft Failure by HLA Antibodies: A Prospective Trial. Am J Transplant 4(3): 438-443. 
4. Hoshino J, Everly MJ, Kaneku H, Ubara Y, Takaichi K, et al. (2014) Impact of the presence and duration of donor-specific antibodies on renal function. Transplant Proc 46(1): 75-80.

5. Irure J, López-Hoyos M, Rodrigo E, Gómez-Román J, Ruiz JC, et al. (2016) Antibody-Mediated Rejection in Kidney Transplantation Without Evidence of Anti-HLA Antibodies? Transplant Proc 48(9): 2888-2890.

6. Locke AF, Zhang Q Reed EF, Gjertson DW (2016) OR7 The UCLA virtual crossmatch exchange. Hum Immunol 77: 7.

7. Jani V, Ingulli E, Mekeel K, Morris GP (2016) Root cause analysis of limitations of virtual crossmatch for kidney allocation to highlysensitized patients. Hum Immunol 78(2): 72-79.

8. Tambur AR, Ramon DS, Kaufman DB, Friedewald J, Luo X, et al. (2009) Perception versus reality?: Virtual crossmatch - How to overcome some of the technical and logistic limitations. Am J Transplant 9(8): 18861893.

9. Morris GP, Phelan DL, Jendrisak MD, Mohanakumar T (2010) Virtual crossmatch by identification of donor-specific anti-human leukocyte antigen antibodies by solid-phase immunoassay: A 30-month analysis in living donor kidney transplantation. Hum Immunol 71(3): 268-273.

10. Piazza A, Ozzella G, Poggi E, Caputo D, Manfreda A, et al. (2014) Virtual Crossmatch in Kidney Transplantation. Transplant Proc 46(7): 21952198.

11. Sullivan HC, Liwski RS, Bray RA, Gebel HM (2017) The Road to HLA antibody evaluation: Do not rely on MFI. Am J Transplant 17(6): 14551461.

12. Salvadé I, Aubert V, Venetz JP, Golshayan D, Saouli AC, et al. (2016) Clinically-relevant threshold of preformed donor-specific anti-HLA antibodies in kidney transplantation. Hum Immunol 77(6): 483-489.

13. Vivek J, Barry F, Chunlong Y, Gerald M (2016) P131 Limitations of virtual crossmatch for deceased kidney transplantation in highly sensitized patients. Hum Immunol 77: 132.

14. Gebel HM, Bray RA (2014) HLA antibody detection with solid phase assays: Great expectations or expectations too great? Am J Transplant 14(9): 1964-1975.

15. Jucaud V, Ravindranath MH, Terasaki PI (2017) Conformational Variants of the Individual HLA-I Antigens on Luminex Single Antigen Beads Used in Monitoring HLA Antibodies: Problems and Solutions. Transplantation 101(4): 764-777.

16. Gebel HM, Liwski RS, Bray RA (2013) Technical aspects of HLA antibody testing. Curr Opin Organ Transplant 18(4): 455-462.

17. Sullivan HC, Gebel HM, Bray RA (2017) Understanding solid-phase HLA antibody assays and the value of MFI. Hum Immunol 78(7-8): 471-480.

18. Wasik C, Dastych K, Sun W, Chen J, Gaitonde S (2017) P117 Limitation of donor specific antibody mean florescent intensity values in predicting transplant compatibility: The saga continues. Hum Immunol 78: 139.

19. Adebiyi 00, Gralla J, Klem P, Freed B, Davis S, et al. (2016) Clinical Significance of Pretransplant Donor-Specific Antibodies in the Setting of Negative Cell-Based Flow Cytometry Crossmatching in Kidney Transplant Recipients. Am J Transplant 16(12): 3458-3467.

20. Ho EK, Vasilescu ER, Colovai AI, Stokes MB, Hallar M, et al. (2008) Sensitivity, specificity and clinical relevance of different cross-matching assays in deceased-donor renal transplantation. Transpl Immunol 20(12): 61-67.

21. Amrouche L, Aubert O, Suberbielle C, Rabant M, Duong J-P, et al. (2017) Long-term outcomes of kidney transplantation in patients with high levels of preformed DSA: the Necker high-risk transplant program. Transplantation 101(10): 2440-2448.
Creative Commons Attribution 4.0 International License

For possible submissions Click Here
Submit Article

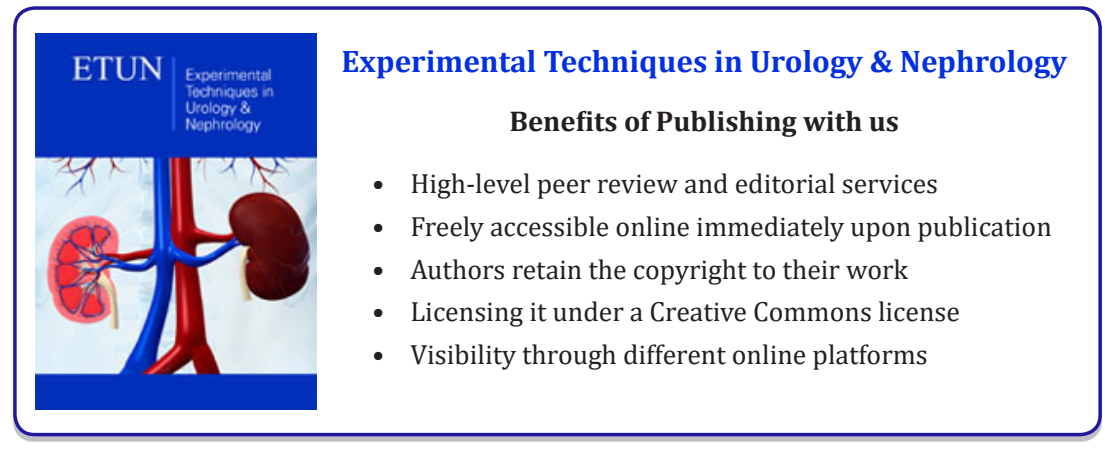

